\title{
Performance based Analysis of Wavelets Family for Image Compression-A Practical Approach
}

\author{
Neeraj Saini \\ M.Tech Student, Computer Science, Krishna \\ Engineering College, Ghaziabad, U.P.
}

\author{
Pramod Sethy \\ Assistant Professor, CSE, Krishna Engineering \\ College, Ghaziabad, U.P.
}

\begin{abstract}
Image compression has become vital in today's scenario because lots of digital images has become spread over the servers which uses lots of space. The size of image files increased in the past 15 years due to the rapid technological advancement and increasing quality of images. In such conditions, it has become significant to use an efficient algorithm to compress images in such a manner so that it gives better compression with minimum loss in quality with respect to different quality measures. The proposed image compression method is implemented in frequency domain using wavelet transform. In this paper, Haar, Symlet, Coiflets, reverse biorthogonal, biorthogonal, and Daubechies wavelets are analyzed for the compression. There are mother wavelets, each of them is analyzed with respect to image compression scheme and resulting wavelets are given as the output which wavelets are resulting in goodcompression.
\end{abstract}

\section{Keywords}

Discrete Wavelet Transform, Haar, Symlet, Coiflets, orthogonal, bi-orthogonal, Daubechies wavelets, MSE, PSNR, Universal Quality Index, NCC, NAE, Structural Content

\section{INTRODUCTION}

An image in general may be defined as a 2-D function $\mathrm{f}(\mathrm{x}, \mathrm{y})$, where $\mathrm{x}, \mathrm{y}$ are spatial (plane) coordinates, and the amplitude of function $\mathrm{f}$ at any pair of coordinates $(\mathrm{x}, \mathrm{y})$ is called the intensity or pixel value of the image at that point. When $\mathrm{x}, \mathrm{y}$, and the intensity values of $\mathrm{f}$ are all finite, discrete quantities, called the image a digital image. Digital image processing refers to processing digital images by means of a digital computer. Note that a digital image is composed of a finite number of elements, each of which has a particular location and value. Pixel is the term most widely used to denote the elements of a digital image. Image compression method that employ different encoding, different mask or filter, similarly wavelet transform have been successful in giving high rate of compression while maintaining the good quality. In this paper, the main objective is to suggest a hybrid method for image compression based on successfully implemented tool in matlab and analyzing these wavelet based on generated result. Proposed method is basically a) input an image, b) compress this image by using the tool using different wavelets and cosine transformation to create a compressed file, C) Decompress the compressed file using cosine transformation,d) compare the quality of all images using different quality parameters. In particular, proposed objective is to find the best wavelet according to different quality parameters.

\section{LITERATURE SURVEY}

Navita Palta, Neha Sharma [1] proposed a method of Image Encryption and Compression using HAAR and COIFLET Wavelet Transform. In this work, theyuses lossy compression method, the method applied for compression to encrypted image that proved more efficient in terms of Compression Ratio , Mean Square Error, Peak Signal to Noise Ratio , Entropyand Bit Error Rate.

Tanveer Sultana [2] proposed an algorithm for image compression for different wavelet codes which is the wavelet techniques used for compression of gray scale and true color images. Image compression methods is applied on the EZW has applied to provide high compression rates maintaining ratio can be reached to good quality of the image with different level wavelet codes.

Navneet Kaur Kang, Sanyam Anand [3] proposed a methodology for encryption and compression using Haar and Symlet wavelet transform. Their proposed scheme was Encryption then Compression of medical images where Haar and Symlet wavelets can be applied to efficiently compress the encrypted images. The proposed encryption technique is used on the basis of Prediction Error Clustering and Random Permutation to provide the high security at the transmission and the compression of the encrypted image is operated by the Haar and Symlet wavelets.

Rahul Samnotra, Randheer Singh[4] provides a methodology Effect of Image Compression on Medical Scans Using Wavelet Transform; In this work, medical image has been compressed using different wavelet transforms with different levels of compression. PSNR and MSE of the decomposed images were calculated. PSNR is used to measure the difference between two images. From the several types of wavelet transforms, Haar, Daubechies (db), symlet, and coiflet wavelet transforms were used to analyze the results.

S. Vimla, P. Usha Rani, J. Anitha Joseph [5] proposed A Hybrid Approach to Compress Still Images using Wavelets and Vector Quantization; Vector Quantization is applied after decomposition of image for the lower sub band. VQ is a lossy image compression technique used to have improved coding efficiency followed by different types of wavelets. With all the wavelets, the proposed technique leads to better compression ratio without losing the visual effect.

Prerna Rajput, Shiv kumar[6] proposed Development of Novel Denoising Technique usingTotal Variation and Symlet Wavelet Filter; the denoising of images is novel techniques used in the various multimedia devices noises are collectivelyformulated as Additive White Gaussian Noises (AWGN). In thispaper such scenarios are considered and developed a denoisingtechnique which significantly improves the performance. Theproposed methodology considered Total Variation Model followedby Symlet Filter Decomposition with Hard threshold.

Varinderjit Kaur, Anamika Pathania, Kiran Bala[7] proposed the analysis of Fast wavelet Transformation; the objective of this paper is to evaluate a set of wavelets for image compression. Image compression using wavelet transforms 
results in better-quality compression ratio. Here in this paper they examined and compared various wavelet families such as Haar, Symlets and Biorthogonal using Discrete Wavelet Transform and Fast wavelet transform. The study compares DWT and FWT approach in terms of PSNR, Compression Ratios and elapsed time for several Images. Complete analysis is performed at second and third level of decomposition

Reeta Charde, Neha Choudhary, Anand Mantri [8] analyses Compression Effect on Images using different Wavelet Transformation; Wavelet transforms are specially used for compression, Denoising, Thresholding, Error reduction, reconstruction, and for image synthesis. Huffman coding can be used for the quantization depending on the method. More sophisticated methods are available which combine wavelet decomposition and quantization. There are different types of wavelets are used for image and data compression. Each level has different compression ratio and bits per pixel (BPP) information. On selecting a particular level Mean Square Error (MSE) as well as Peak Signal to Noise Ratio (PSNR) will change. The challenge of compression methods is to find the best compromise between a low compression ratio and a good perceptual result.

Priyatosh Halder[9] proposed Hadamard-Coiflets transform with arithmetic coding to increase the visual quality of image. The improved Hadamard-Coiflets transform with arithmetic coding is good techniques of compression and can able to give higher PSNR value as compared to various existing methods. Hadamard-Coiflets transform is applied and then on each block of the low frequency subband and split all values from each transformed block followed by applying arithmetic coding for image compression.

J. L.Shrinivas, P Sunita,[10] proposed performance analysis of SPIHT algorithm in image compression This is a technique for compression of gray scale and color images using SPIHT algorithm along with wavelets. Compression can be done using different wavelets at different levels of decomposition. The performance analysis of SPIHT with bi-orthogonal wavelets at four different levels of decomposition using EZW coding was presented. The technique performance of SPIHT compression is compared in terms of PSNR, MSE, BPP, and $\mathrm{CR}$

S. Shridhar, P. Rajesh Kumar, K.V.Ramanaiah[11] proposed the analysis to choosing wavelet for image compression; The choice of wavelet family depends on the application and the content of image. Proposed work is carried out by the application of different wavelet families. In this paper the relative merits of different Wavelet transform techniques are evaluated using quality measures PSNR and MSE,

Nitin Sharma, MayankSingh [12] proposed an algorithm in Wavelets Domain for Direct Application to Revolutionize, Modeling and Imaging Technique through Crossbreeding Wavelet and Cosine Transformation. Lots of technique are proposing with different method or tool, here compression technique are proposing with the help of cosine transformation and wavelets transformations with , $\mathrm{db} 7^{\mathrm{ce}}$, „db7 $7^{\text {ee }}$ and „db8 $8^{\text {ee }}$. And calculate the compression ratio using crossbreed architecture and wavelets , $\mathrm{db} 7^{\mathrm{ee}},, \mathrm{db} 7^{\mathrm{ec}}$ and , $\mathrm{db} 8^{\mathrm{ec}}$ and in the last try to find out which transformation combination is best with respect to compression ratio.

\section{WAVELET TRANSFORM}

The uses of Wavelet Transform is in image processing such as image compression, edge detection, noise removal .Wavelets are mathematical tool for shifting the coordinate system in which signal represent to another domain that is suitable for compression. Wavelet based coding is verydynamic under transmission and decoding errors. Wavelets are method for decomposing signals such as image, into an order of increasing resolution. A wavelet transform can be used to decompose a signal into component wavelets. Wavelets have great benefit of being able to distinct the time details. Wavelet is a small wave whose energy is concentrated in time and all these function are created from a single function called mother wavelet by expansions and translation in time domain. Wavelet produces a natural multi resolution of every image including the all-important edges. Wavelets are signals which are local in scale and time and generally have an irregular shape. A wavelet is a waveform of effectively limited period that has an average value of zero. Mathematically, the wavelet is a function of zero average, having the energy focused in time:

$$
\int_{-\infty}^{\infty} \Psi(t)=0
$$

The term wavelet comes from the fact that they integrate to zero and they wave up and down across the axis. A signal can be decomposed into many shifted and scaled representation of the original mother wavelet. In order to be more accurate in extracting time and frequency information, a family of Wavelets can be constructed from a function ( $t$ ), also known as the Mother Wavelet, in a finite interval. Daughter Wavelets are then formed by translation with a factor and dilation with a scale parameter s:

$$
\begin{gathered}
\Psi_{u, s}(t)=\frac{1}{\sqrt{s}} \Psi\left(\frac{t-u}{s}\right) \text { or } \\
h_{a, b}(t)=\frac{1}{\sqrt{a}} h\left(\frac{t-b}{a}\right)
\end{gathered}
$$

\section{DISCRETE WAVELET TRANSFORM}

In two dimensional discrete wavelet transform, digital data is decomposed in four frequency sub-bands. Discrete wavelet transform decompose the original cover image into four frequency sub-bands namely LL, HH, LH and HL. Here (fig 1 and fig 2) LL frequency sub-band establishes the estimate details. The frequency sub-band LH is used to constitute the vertical details of the image, HL contains the horizontal details of the image and the $\mathrm{HH}$ sub-band contains the diagonal details of the image. The LL sub-band that is the approximation of the digital image could be further decomposed with the use of discrete wavelet transform to get any level of decomposition of the digital content and it will generate the further four sub-bands. Thus multiple levels of decomposition could be obtained by applying the discrete wavelet transform on the approximation part, that is, on the LL part of the digital content as desired by the application. 


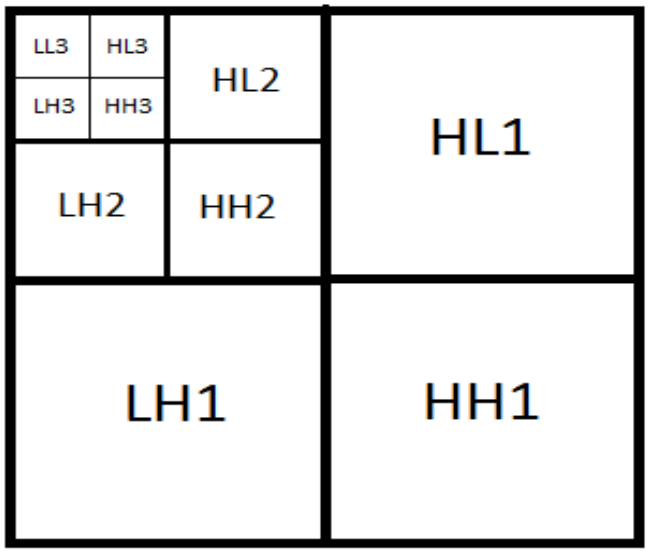

Fig 1: Decomposition of image at 3rd level using wavelet

These sub band are the decomposition of original image. Sub band LL caries approximate element of image, LH contain the vertical element of image, HL contain the horizontal element of image and $\mathrm{HH}$ contains diagonal element of image. Thus the information of image is stored in decomposed form in these sub bands.

Wavelet transform deals with both the spatial and frequency correlation of data by dilations (or contractions) and translations of mother wavelet on input data. It can be applied to different scales according to the details required, which allows progressive transmission and zooming of the image without the need of extra storage.The wavelet transform has gained extensive acceptance in signal processing and image compression. Because of their intrinsic multi-resolution nature, wavelet-coding schemes are mainly suitable for applications where scalability and tolerable degradation are significant.

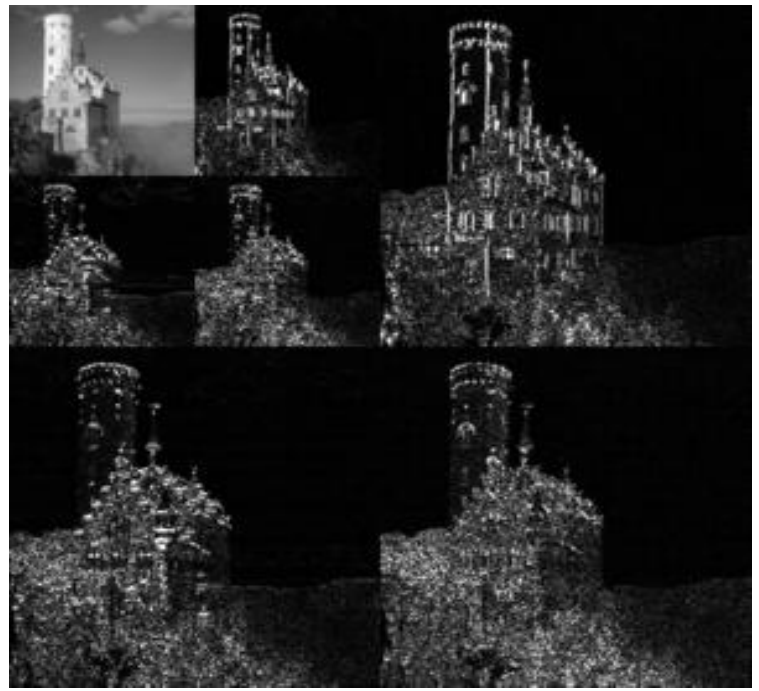

Fig 2: 3rd level of decomposition of an image

Wavelet transform decomposes a signal into a set of basic functions. These basis functions are called wavelets which are obtained by dilations and shifting from a single prototype wavelet called mother wavelet.

\section{WAVELET FAMILIES DESCRIPTION}

Wavelet family is the family of similar types of wavelets. The main wavelet of the family is called mother wavelet. Some of wavelet family are named below:

\subsection{Haar wavelet}

It is based on a class of orthogonal matrices whose elements are $1,-1$, or 0 . Disadvantage of this wavelet is not continuous \& not differentiable.

\subsection{Daubechies wavelets}

This family is based on orthogonal, and categorized by supported scaling wavelet functions, which generates an orthogonal multi-resolution analysis. This wavelet function is denoted as db1. It is difficult to get an orthogonal supported wavelet that is either symmetric or asymmetric except for Haar wavelets.

\subsection{Coiflets}

It is same as daubechies and maximal number of vanishing moments and the scaling function form $2 \mathrm{~N}-1$ moment equal to 0 . And this general wavelet function has $2 \mathrm{~N}$ moments equal to 0 . The two function support of length $6 \mathrm{~N}-1$.

\subsection{Symlet}

They are based on least asymmetric and maximum number of vanishing moments. They are called as symmetric wavelets.

\subsection{Biorthogonal}

They are denoted as bior wavelet, biorthogonal if often used instead of orthogonal i.e. rather than having one scaling and wavelet function, there are two scaling functions that may generate different multi-resolution analysis, and accordingly two different wavelet functions used in the analysis and combination.

\subsection{Reverse Biorthogonal}

It is based on reconstruction and decomposition of scaling filters. This wavelet has vanishing moments on decomposition for analysis and vanishing moment for the reconstruction of synthesis. It is denoted as rbio.

\subsection{Discrete FIR Meyer wavelet}

It is based on symmetric, orthogonal and biorthogonal. It is denoted by demay.

\section{THE PROPOSED ALGORITHM}

Basically cosine transformation had applied for image compression with JPEG and individually wavelets transformation can also apply for compression purpose. Here a crossbreeding approach are applying for compression purpose.Steps of algorithm are as follows:

Step 1:Input a bitmap image (grayscale for fast compression).

Step2: Decompose image at second level with the help of wavelet transformation

Step3: Apply cosine transformation on resulting $8 * 8$ block

Step4: Apply quantization and sampling on each block.

Step5: Save the compressed image into unreadable format.

Step6: Fetch the compressed image file.

Step7: Apply de-sampling and de-quantization on each block.

Step8: Apply inverse cosine transformation on resulting matrix.

Step9:Combined the blocks followed apply inverse wavelets transformation.

Step10: Save the decompressed image in bitmap format. 
Step11. Calculate different quality parameter for each wavelet.

Step 12. Calculate different quality parameters for decompressed image.

\section{EXPERIMENTAL RESULTS}

Table 1 shows the effect of different wavelets on a bmp image.By analyzing this table it can be understand that which wavelet gives higher compression ratio.

Table 1: Analysis of wavelets

\begin{tabular}{|c|c|c|c|c|}
\hline $\begin{array}{l}\text { USED } \\
\text { WAVELE } \\
\text { T }\end{array}$ & $\begin{array}{c}\text { ORIG } \\
\text { INAL } \\
\text { IMAG } \\
\text { E } \\
\text { SIZE } \\
\text { (IN } \\
\text { MB)A } \\
\end{array}$ & $\begin{array}{c}\text { COMP } \\
\text { RESSE } \\
\text { D } \\
\text { FILE } \\
\text { SIZE } \\
\text { (IN } \\
\text { KB)B } \\
\end{array}$ & $\begin{array}{c}\text { DECOM } \\
\text { PRESSE } \\
\text { D } \\
\text { IMAGE } \\
\text { SIZE (IN } \\
\text { MB) } \\
\end{array}$ & $\begin{array}{c}\text { Compressi } \\
\text { on ratio } \\
\left(1-\frac{B}{A}\right)\end{array}$ \\
\hline dmey & 3.34 & 202 & 3.35 & 94.09384 \\
\hline coif1 & 3.34 & 109 & 3.35 & 96.81301 \\
\hline coif 2 & 3.34 & 111 & 3.34 & 96.75454 \\
\hline coif3 & 3.34 & 111 & 3.35 & 96.75454 \\
\hline coif4 & 3.34 & 119 & 3.34 & 96.52063 \\
\hline coif5 & 3.34 & 118 & 3.35 & 96.54987 \\
\hline bior1.1 & 3.34 & 106 & 3.35 & 96.90073 \\
\hline bior1.3 & 3.34 & 105 & 3.35 & 96.92997 \\
\hline bior1.5 & 3.34 & 111 & 3.35 & 96.75454 \\
\hline bior2.2 & 3.34 & 101 & 3.35 & 97.04692 \\
\hline bior 2.4 & 3.34 & 106 & 3.35 & 96.90073 \\
\hline bior 2.6 & 3.34 & 106 & 3.35 & 96.90073 \\
\hline bior 2.8 & 3.34 & 113 & 3.35 & 96.69606 \\
\hline bior3.1 & 3.34 & 77 & 3.34 & 97.74864 \\
\hline bior3.3 & 3.34 & 88 & 3.34 & 97.42702 \\
\hline bior3.5 & 3.34 & 96 & 3.34 & 97.19311 \\
\hline bior3.7 & 3.34 & 96 & 3.34 & 97.19311 \\
\hline bior3.9 & 3.34 & 104 & 3.34 & 96.95921 \\
\hline bior4.4 & 3.34 & 103 & 3.35 & 96.98845 \\
\hline bior5.5 & 3.34 & 95 & 3.34 & 97.22235 \\
\hline bior6.8 & 3.34 & 113 & 3.35 & 96.69606 \\
\hline rbio1.1 & 3.34 & 106 & 3.35 & 96.90073 \\
\hline rbio1.3 & 3.34 & 99 & 3.35 & 97.1054 \\
\hline rbio1.5 & 3.34 & 102 & 3.35 & 97.01768 \\
\hline rbio2.2 & 3.34 & 114 & 3.35 & 96.66682 \\
\hline rbio2.4 & 3.34 & 106 & 3.35 & 96.90073 \\
\hline rbio2.6 & 3.34 & 117 & 3.35 & 96.57911 \\
\hline rbio2.8 & 3.34 & 118 & 3.35 & 96.54987 \\
\hline rbio3.1 & 3.34 & 117 & 3.34 & 96.57911 \\
\hline rbio3.3 & 3.34 & 107 & 3.34 & 96.87149 \\
\hline rbio3.5 & 3.34 & 108 & 3.34 & 96.84225 \\
\hline rbio3.7 & 3.34 & 109 & 3.34 & 96.81301 \\
\hline
\end{tabular}

\begin{tabular}{|l|l|l|l|l|}
\hline rbio3.9 & 3.34 & 112 & 3.34 & 96.7253 \\
\hline rbio4.4 & 3.34 & 107 & 3.35 & 96.87149 \\
\hline rbio5.5 & 3.34 & 96 & 3.34 & 97.19311 \\
\hline rbio6.8 & 3.34 & 115 & 3.35 & 96.63758 \\
\hline sym2 & 3.34 & 97 & 3.34 & 97.16388 \\
\hline sym3 & 3.34 & 92 & 3.35 & 97.31007 \\
\hline sym4 & 3.34 & 105 & 3.34 & 96.92997 \\
\hline sym5 & 3.34 & 99 & 3.35 & 97.1054 \\
\hline sym6 & 3.34 & 110 & 3.34 & 96.78378 \\
\hline sym7 & 3.34 & 106 & 3.35 & 96.90073 \\
\hline sym8 & 3.34 & 112 & 3.34 & 96.7253 \\
\hline sym10 & 3.34 & 115 & 3.34 & 96.63758 \\
\hline sym13 & 3.34 & 110 & 3.35 & 96.78378 \\
\hline sym15 & 3.34 & 122 & 3.35 & 96.43292 \\
\hline sym17 & 3.34 & 118 & 3.35 & 96.54987 \\
\hline sym19 & 3.34 & 132 & 3.35 & 96.14053 \\
\hline sym20 & 3.34 & 125 & 3.35 & 96.3452 \\
\hline sym21 & 3.34 & 126 & 3.35 & 96.31596 \\
\hline sym23 & 3.34 & 141 & 3.35 & 95.87739 \\
\hline sym25 & 3.34 & 136 & 3.35 & 96.02358 \\
\hline sym27 & 3.34 & 147 & 3.35 & 95.70196 \\
\hline sym30 & 3.34 & 153 & 3.34 & 95.52653 \\
\hline sym31 & 3.34 & 157 & 3.35 & 95.40957 \\
\hline haar & 3.34 & 106 & 3.35 & 96.90073 \\
\hline db2 & 3.34 & 97 & 3.35 & 97.16388 \\
\hline db4 & 3.34 & 94 & 3.35 & 97.25159 \\
\hline db6 & 3.34 & 104 & 3.35 & 96.95921 \\
\hline db8 & 3.34 & 113 & 3.35 & 96.69606 \\
\hline db10 & 3.34 & 135 & 3.35 & 96.05282 \\
\hline db20 & 3.34 & 202 & 3.35 & 94.09384 \\
\hline
\end{tabular}

This table gives the compression ratio for different wavelet compression, this denotes that all mother wavelets are independent with respect to compression. The size of resulting decompressed image is same as input image but quality is different. Further some wavelets are reduced from table which gives worse quality according to look and feel then the decompressed image is resized equal to original image's dimension for comparing the quality loss in decompressed image and thus applied different quality parameter which are as follows:

\subsection{Quality Parameters}

There are some quality parameters [13] on the basis of which, deviation between the original image and the resultant image is calculated. These parameter are as follows:

\subsubsection{MSE}

The original image A of $\mathrm{m}^{*} \mathrm{n}$ and Decompressed image B of same dimension is given then Mean Square Error can be define as following: 


$$
M S E=\frac{1}{m n} \sum_{i=0}^{m-1} \sum_{j=0}^{n-1}[A(i, j)-B(i, j)]^{2}
$$

The ideal value for MSE is zero.

\subsubsection{MAX}

Maximum Difference should be 0 for same images but if images are different then often it reaches to maximum value i.e. 255 and it can be expressed as follows:

$$
\mathrm{MD}=\operatorname{Max}\left(\mid \mathrm{A}_{\mathrm{ij}}-\mathrm{B}_{\mathrm{ij}}\right)
$$

Where $i=1,2, \ldots, m$ and $j=1,2, \ldots, n$

\subsubsection{PSNR}

PSNR stands for Peak Signal-to-Noise Ratio and its ideal value is infinity and it can be expressed as follows:

$$
\begin{gathered}
P S N R=10 \cdot \log _{10}\left(\frac{M A X^{2}}{M S E}\right) \\
P S N R=20 \cdot \log _{10}\left(\frac{M A X}{\sqrt{M S E}}\right) \\
P S N R=20 \cdot \log _{10} M A X-10 \cdot \log _{10} M S E
\end{gathered}
$$

\subsubsection{NormalizedCross Correlation}

Normalizedcross Correlation can be expressed as follows:

$$
N C C=\sum_{i=1}^{m} \sum_{i=1}^{n} \frac{A_{i j} * B_{i j}}{A_{i j}^{2}}
$$

The ideal value of Normalized cross correlation is 1 .

\subsubsection{Normal Absolute Error}

Normalized Absolute Error should be 0 and it can be defined as follows:

$$
N A E=\frac{\sum_{i=1}^{m} \sum_{j=1}^{n}\left(\left|A_{i j}-B_{i j}\right|\right)}{\sum_{i=1}^{m} \sum_{j=1}^{n}\left(A_{i j}\right)}
$$

\subsubsection{Structural Content}

The structural content should be 1 ideally and it can be defined as follows:

$$
S C=\frac{\sum_{i=1}^{m} \sum_{j=1}^{n}\left(A_{i j}{ }^{2}\right)}{\sum_{i=1}^{m} \sum_{j=1}^{n}\left(B_{i j}{ }^{2}\right)}
$$

\subsubsection{Universal quality index}

Universal quality index [14] is the new parameter for comparison of quality of the image. Let $x=\{x i \mid i=1,2, \ldots, N\}$ and $y=\{y i \mid i=1,2, \ldots, N\}$ be the original and the test image(i.e. Decompressed image) signal respectively. The quality index $\mathrm{Q}$ is defined as:

$$
\begin{gathered}
Q=\frac{4 \sigma_{x y} \bar{x} \bar{y}}{\left(\sigma_{x}{ }^{2}+{\sigma_{y}}^{2}\right)\left[(\bar{x})^{2}+(\bar{y})^{2}\right]} \\
\text { Where, } \bar{x}=\frac{1}{N} \sum_{i=1}^{N} x_{i}, \quad \bar{y}=\frac{1}{N} \sum_{i=1}^{N} y_{i} \\
\sigma_{X}^{2}=\frac{1}{N-1} \sum_{i=1}^{N}\left(x_{i}-\bar{x}\right)^{2}, \quad \sigma_{y}^{2}=\frac{1}{N-1} \sum_{i=1}^{N}\left(y_{i}-\bar{y}\right)^{2} \\
\sigma_{x y}=\frac{1}{N-1} \sum_{i=1}^{N}\left(x_{i}-\bar{x}\right)\left(y_{i}-\bar{y}\right)
\end{gathered}
$$

The range of $\mathrm{Q}$ is $[-1,1]$. Theideal value $\mathrm{Q}=1$ will achieve iff $y i=x i$ for all $i=1,2, \ldots, N$, i.e. both images are same.

After compression some wavelets are selected which are giving better image according to look and feel then analyze different quality parameter with the help of used tool in MATLAB (fig 3) to observe the decrease in quality after lossy compression also to find the better result giving wavelet. The result of the proposed compression scheme for the wavelets can be seen in table 2 .

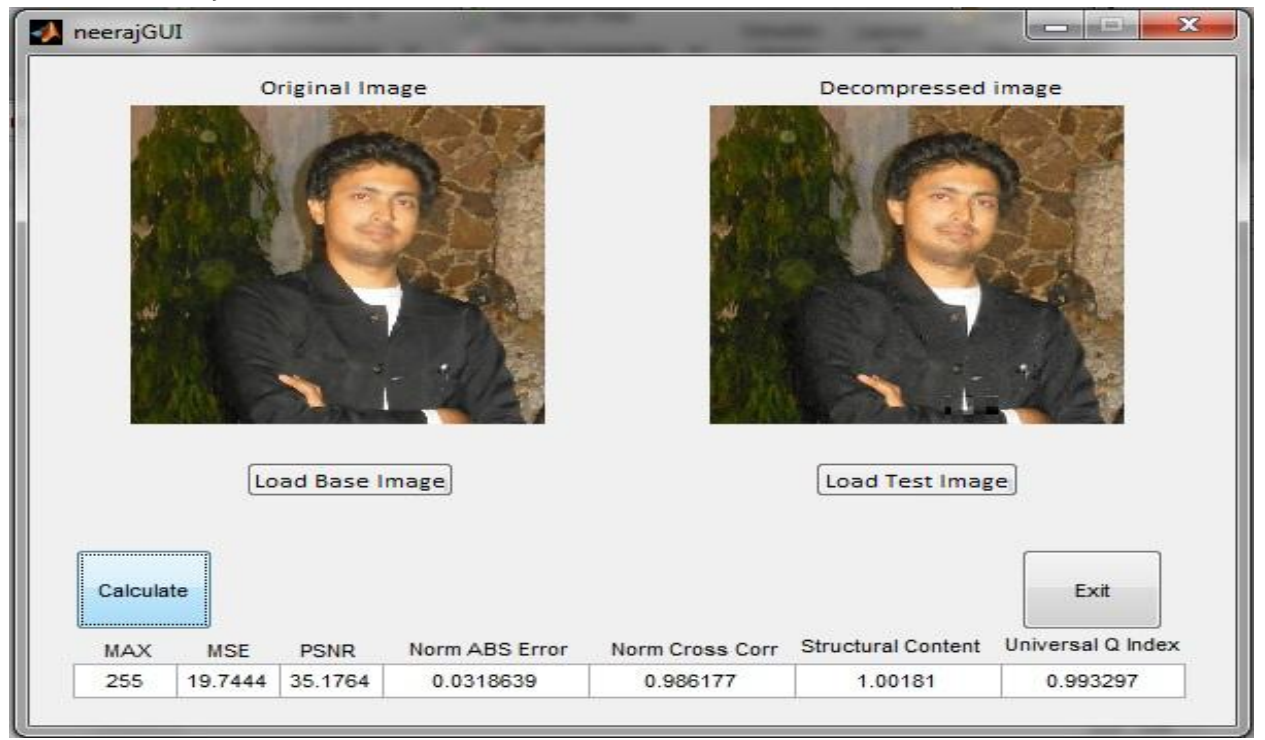

Fig 3: Tool for calculating quality parameter

Table 2: Different quality parameter value after comparing original image and decompressed image using wavelets 


\begin{tabular}{|c|c|c|c|c|c|c|c|}
\hline Wavelet & $\begin{array}{l}\text { Maximum } \\
\text { Difference }\end{array}$ & MSE & PSNR & $\begin{array}{c}\text { Normal } \\
\text { Absolute } \\
\text { Error } \\
\end{array}$ & $\begin{array}{c}\text { Structural } \\
\text { Content }\end{array}$ & $\begin{array}{c}\text { Normal Cross } \\
\text { Correlation }\end{array}$ & $\begin{array}{c}\text { Universal Quality } \\
\text { Index }\end{array}$ \\
\hline bior1.3 & 255 & 39.1538 & 32.2031 & 0.0375619 & 1.00012 & 0.967132 & 0.973758 \\
\hline bior1.5 & 255 & 41.4243 & 31.9582 & 0.0394744 & 1.00116 & 0.965016 & 0.979296 \\
\hline bior4.4 & 255 & 28.9558 & 33.5134 & 0.0301174 & 0.999848 & 0.974552 & 0.979872 \\
\hline bior5.5 & 255 & 17.6971 & 35.6518 & 0.0181167 & 0.999482 & 0.991828 & 0.987111 \\
\hline bior6.8 & 255 & 25.055 & 34.1419 & 0.0280237 & 0.998389 & 0.973689 & 0.976447 \\
\hline coif1 & 255 & 35.5133 & 32.6269 & 0.0348464 & 0.999338 & 0.97186 & 0.974538 \\
\hline coif2 & 255 & 31.4098 & 33.1602 & 0.0291716 & 1.00097 & 0.980484 & 0.984666 \\
\hline coif3 & 255 & 33.8775 & 32.8317 & 0.0336323 & 1.00112 & 0.972063 & 0.974771 \\
\hline coif4 & 255 & 30.2525 & 33.3232 & 0.0282874 & 1.00204 & 0.980705 & 0.982645 \\
\hline coif5 & 255 & 30.3965 & 33.3026 & 0.0311444 & 1.00094 & 0.973726 & 0.976099 \\
\hline rbio1.1 & 255 & 36.1574 & 32.5488 & 0.034808 & 0.99721 & 0.96901 & 0.977667 \\
\hline rbio1.3 & 255 & 29.9684 & 33.3642 & 0.0304487 & 0.999793 & 0.972403 & 0.970618 \\
\hline rbio1.5 & 255 & 28.6605 & 33.558 & 0.0294107 & 0.999389 & 0.973282 & 0.975433 \\
\hline rbio2.2 & 255 & 32.8359 & 32.9673 & 0.0325002 & 0.999574 & 0.971979 & 0.975565 \\
\hline rbio2.4 & 255 & 38.9923 & 32.221 & 0.0378486 & 1.0019 & 0.967411 & 0.971077 \\
\hline rbio2.6 & 255 & 39.2516 & 32.1922 & 0.037681 & 1.00176 & 0.968434 & 0.971107 \\
\hline rbio2.8 & 255 & 37.8881 & 32.3458 & 0.0369066 & 1.00256 & 0.968283 & 0.973565 \\
\hline rbio4.4 & 255 & 36.2159 & 32.5418 & 0.0355996 & 1.00139 & 0.970073 & 0.975597 \\
\hline rbio5.5 & 255 & 17.6167 & 35.6716 & 0.0176628 & 0.999407 & 0.991355 & 0.989003 \\
\hline rbio6.8 & 255 & 34.6759 & 32.7305 & 0.0343705 & 1.0017 & 0.97108 & 0.972312 \\
\hline sym2 & 255 & 29.1281 & 33.4877 & 0.0280439 & 1.00066 & 0.97962 & 0.980944 \\
\hline sym3 & 255 & 32.7001 & 32.9853 & 0.0330045 & 1.00088 & 0.972016 & 0.969466 \\
\hline sym4 & 255 & 29.8199 & 33.3857 & 0.0281861 & 1.00096 & 0.980693 & 0.983051 \\
\hline sym5 & 255 & 29.8453 & 33.382 & 0.0307708 & 0.999991 & 0.973983 & 0.974915 \\
\hline sym6 & 255 & 23.7392 & 34.3761 & 0.0227122 & 1.00042 & 0.986796 & 0.988142 \\
\hline sym7 & 255 & 25.4253 & 34.0781 & 0.0284126 & 0.998568 & 0.973419 & 0.974342 \\
\hline sym8 & 255 & 24.3352 & 34.2685 & 0.0230198 & 1.00078 & 0.987082 & 0.985918 \\
\hline sym10 & 255 & 23.9082 & 34.3453 & 0.0227005 & 1.00065 & 0.986894 & 0.983404 \\
\hline sym13 & 255 & 29.3325 & 33.4573 & 0.0304098 & 1.001 & 0.973896 & 0.970773 \\
\hline sym15 & 255 & 30.0863 & 33.3471 & 0.0309315 & 1.00119 & 0.973867 & 0.977675 \\
\hline sym17 & 255 & 29.7838 & 33.391 & 0.0307613 & 1.00117 & 0.97379 & 0.979953 \\
\hline sym19 & 255 & 29.7018 & 33.403 & 0.0305821 & 1.00091 & 0.974101 & 0.976645 \\
\hline sym 21 & 255 & 33.8512 & 32.8351 & 0.033495 & 1.00278 & 0.971643 & 0.977797 \\
\hline sym23 & 255 & 34.8068 & 32.7142 & 0.0342303 & 1.00322 & 0.971129 & 0.972505 \\
\hline sym 25 & 255 & 33.8643 & 32.8334 & 0.0338003 & 1.0028 & 0.97103 & 0.975252 \\
\hline $\operatorname{sym} 27$ & 255 & 33.7914 & 32.8427 & 0.0335308 & 1.0031 & 0.971551 & 0.972099 \\
\hline sym30 & 255 & 27.9001 & 33.6747 & 0.0258733 & 1.00312 & 0.983051 & 0.989961 \\
\hline sym31 & 255 & 34.0755 & 32.8064 & 0.0338568 & 1.00354 & 0.971008 & 0.975648 \\
\hline
\end{tabular}

The above table gives the value of different quality parameter when an image is compressed by using above wavelets.
This table also give basic idea that which wavelet should be use when a particular quality parameter is important. 


\section{CONCLUSION}

Wavelet based compression give good compression ratio without losing too much quality of original image. Quality of image will be deducted only very first time of compression. All mother wavelets are independent with respect to image compression. Higher wavelet of symlets, biorthogonal, and reverse biorthogonal wavelet takes more time. The result of table 2 gives and abstract idea to use appropriate wavelet according to different parameters.If MSE is important factor than rbior5.5 or bior5.5 waveletshould be used, if Quality Index is important then sym30, rbio5.5 or bior5.5, use sym5 wavelets should be used, if structural content and if Compression ratio is important than sym 3 should be used.

\section{REFERENCES}

[1] Navita Palta, Neha Sharma 2015, 'Image encryption and compression using haar and coiflet wavelet transform', International Journal of computer science and information technologies, vol 6(3) 2015.

[2] Tanveer Sultana 2015, 'Image compression algorithm for different wavelet codes', International journal of latest trends in Engineering and technology vol(5) 2015.

[3] Navneet Kaur Kang, Sanyam Anand 2015, 'Encryption then compression system using haar and symlet wavelet transform', International journal of computer technology and applications, vol6 (3), 2015.

[4] Rahul Samnotra, Randhir Singh, 'Effect of Image compression on Medical scan using wavelet transform', International journal of advance research in computer science and software engineering, $\operatorname{vol}(5), 2015$.

[5] Vimla, Usha Rani, Anitha Joseph, 'A hybrid approach to compress still images using wavelet and vector quantization', International journal of engineering and advance technology, vol(4), 2015.

[6] Prerna Rajput, Shiv Kumar, 'Development of novel denoising technique using total variation and symlet wavelet filter', International journal of eengineering trends and technology, vol(22), 2015.
[7] Varinderjit Kaur, Anamika Pathania, Er. Kiran Bala, 'A review paper on advance digital image compression using fast wavelet transforms comparative analysis with DWT', International journal of engineering science and research technologies, 2015.

[8] Reeta Charde, Neha Choudhary, Anand Mantri, 'Compression effect on image using different wavelet transform', International journal of advanced research in Electronics and communication engineering, vol(4), 2015.

[9] Priyatosh Halder, 'An improved Handamart-Coiflet transform for image compression with arithmetic coding, international journal of innovative technology and exploring engineering, $\operatorname{vol}(4), 2015$.

[10] Sunita, L.Srinivas, 'Performance analysis of SPIHT algorithm in image compression',International journal of science, engineering and technology research, vo(4), 2015.

[11] S.Sridhar, Rajesh Kumar, V.Ramanaiah, 'wavelet transform techniques for image compression- an evolution', International journal of image, graphics and signal processing, 2014.

[12] Nitin Sharma, Mayank Singh, 'Image compression tool in wavelets domain for direct application to revolutionize, modeling and imaging technique through crossbreeding wavelet and cosine transformation', International journal of computer application, vol(69), 2013.

[13] Dr.S.S.Bedi, Mrs.Jyoti Agarwal, Pankaj Agarwal, "Image Fusion Techniques and Quality Assessment Parameters for Clinical Diagnosis: A Review", International Journal of Advanced Research in Computer and Communication Engineering Vol. 2, Issue 2, February 2013.

[14] Zhou Wang, Alan C. Bovik,'A Universal Image Quality Index', IEEE Signal Processing Letters, vol XX, no Y, march 2002. 